\title{
Editorial
}

\section{LTE Technology: Antenna, RF Front-Ends, and Channel Modeling}

\author{
Qingfeng Zhang, ${ }^{1}$ Sai Wai Wong, ${ }^{2}$ Cheng Jin, ${ }^{3}$ Xiu Yin Zhang, ${ }^{2}$ Yifan Chen, ${ }^{1}$ \\ Dimitrios Sounas, ${ }^{4}$ and Nima Chamanara ${ }^{5}$ \\ ${ }^{1}$ Department of Electronics and Electrical Engineering, South University of Science and Technology of China, \\ Shenzhen 518055, China \\ ${ }^{2}$ Department of Electronic and Information Engineering, South China University of Technology, Guangzhou 510640, China \\ ${ }^{3}$ Center for Microwave Technology, Beijing Institute of Technology, Beijing 100081, China \\ ${ }^{4}$ Department of Electrical and Computer Engineering, The University of Texas at Austin, Austin, TX 78712, USA \\ ${ }^{5}$ Department of Electrical Engineering, École Polytechnique de Montréal, Montréal, QC, Canada H3T 1J4
}

Correspondence should be addressed to Qingfeng Zhang; zhang.qf@sustc.edu.cn

Received 10 June 2015; Accepted 11 June 2015

Copyright (C) 2015 Qingfeng Zhang et al. This is an open access article distributed under the Creative Commons Attribution License, which permits unrestricted use, distribution, and reproduction in any medium, provided the original work is properly cited.

Mobile communications are more and more involved in our daily life. The long term evolution (LTE) standard is proposed to provide high data rate due to the exploding demands for faster and ubiquitous communication nowadays. It has dramatically driven the technology advances in wireless communication systems. This special issue is aimed at presenting the most recent advances in LTE technology, including the frontend component design and channel modeling techniques. The collected papers represent the state-of-the-art work being carried out in this topic area.

Reducing the antenna size while maintaining a wide bandwidth and high efficiency is highly demanded but also challenging in the design of LTE antennas. The paper by M. Rafiee et al. presents a planar inverted-F antenna (PIFA) used in LTE band 7. They employed interdigital coupling structure to reduce the antenna size and meanwhile to tune the resonant frequency. An interesting benefit of such antenna is that the working frequency can be easily tuned by changing the interdigital part while being able to maintain the size of the whole antenna if required.

Multiple input multiple output (MIMO) technology provides high channel capacity and high data throughput in LTE and the next generation communication systems. However, the compact arrangement of the antennas without mutual coupling remains the most challenging problem in the design of MIMO antennas. The paper by Y. Shi et al. proposes a compact MIMO antenna. The zeroth-order modes of the composite right- and left-hand transmission line (CRLHTL) greatly reduce the size of the antenna. And a pair of L-shaped parasitic strips and an etching slot on the ground are employed to reduce the mutual coupling between two antennas. The paper by I. Tzanidis et al. provides a system level simulation of a 2D active antenna array for FD-MIMO systems. It is found that the proposed antenna array with alternating polarization scheme yields the highest throughput gain. The port virtualization techniques for the antenna array are also discussed in this paper. The paper by $\mathrm{H}$. Shi et al. proposes a direction-of-arrival estimation approach to cope with the scenario where uncorrelated and coherent sources are simultaneously presented in multipath environment. This proposed technique could be potentially applied to MIMO communication.

In addition to antennas, other front-end components, such as filters and power dividers, also exhibit great importance in the efficiency enhancement of LTE communication systems. The paper by W.-Q. Pan et al. presents a dual-band tunable bandpass filter based on a trimode resonator. Another paper by W.-Q. Pan et al. provides a combined solution for power divider and filters, that is, filtering power divider. The integration of filters into other front-end components, for 
example, antennas, amplifiers, and power dividers, greatly reduces the size and meanwhile enhances the performance of the whole system. This topic is getting more and more popular nowadays.

Moreover, this collection includes two papers on channel modeling and interference elimination techniques for LTE communications. The paper by J. F. Monserrat et al. presents a map-based realistic channel model for urban macrocell scenarios, exhibiting a tradeoff between realism and implementation complexity. The paper by $\mathrm{H}$. Zheng et al. carries out an interesting study on the interference problem of the LTE system under radar interference circumstance. They apply the principle of cognitive radio to study the interference between the radar and LTE system and subsequently propose a scheme to get rid of the radar interference.

\title{
Acknowledgments
}

The editors would like to thank all the authors and the anonymous reviewers for their contributions to this special issue.

\author{
Qingfeng Zhang \\ Sai Wai Wong \\ Cheng Jin \\ Xiu Yin Zhang \\ Yifan Chen \\ Dimitrios Sounas \\ Nima Chamanara
}



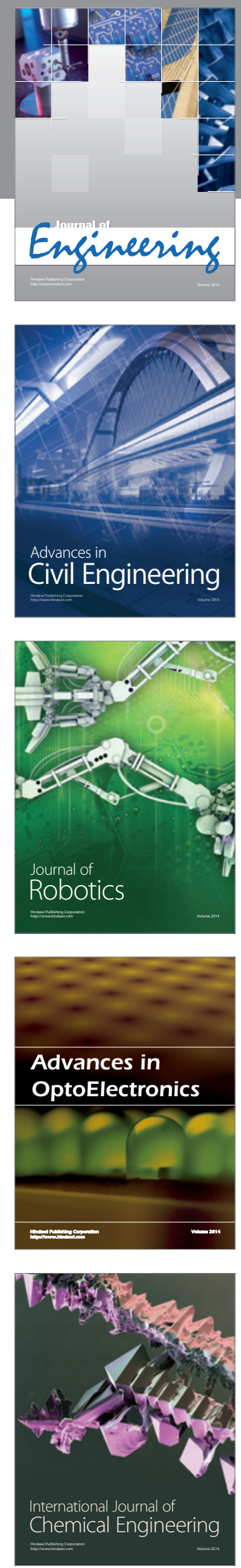

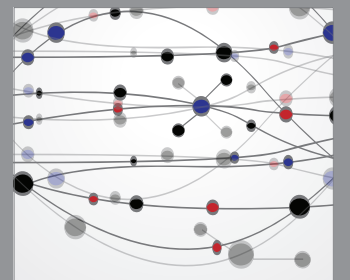

The Scientific World Journal
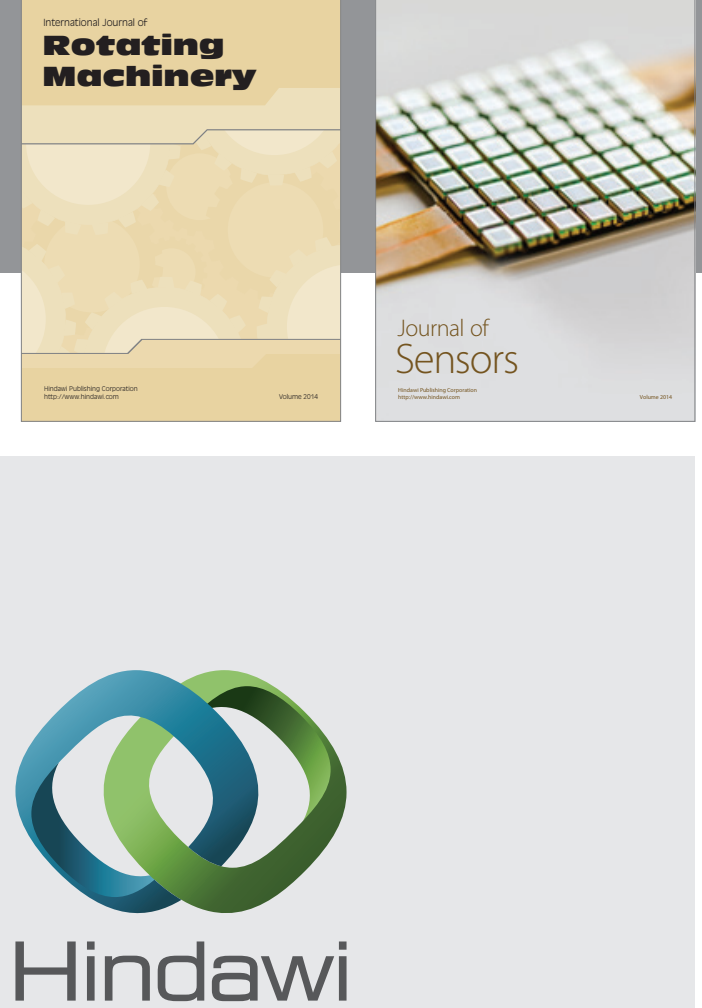

Submit your manuscripts at http://www.hindawi.com
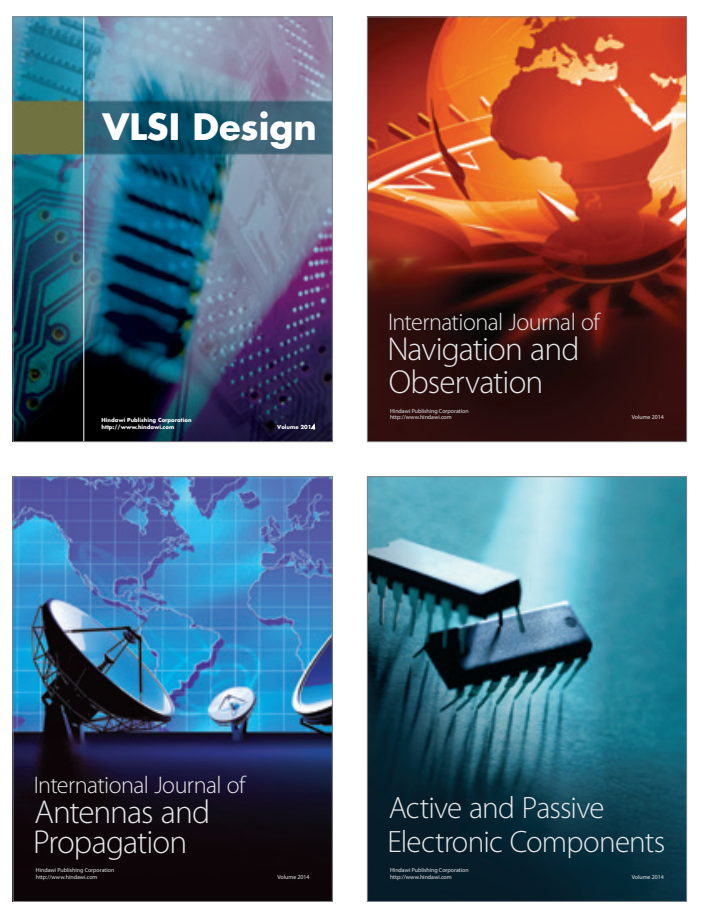
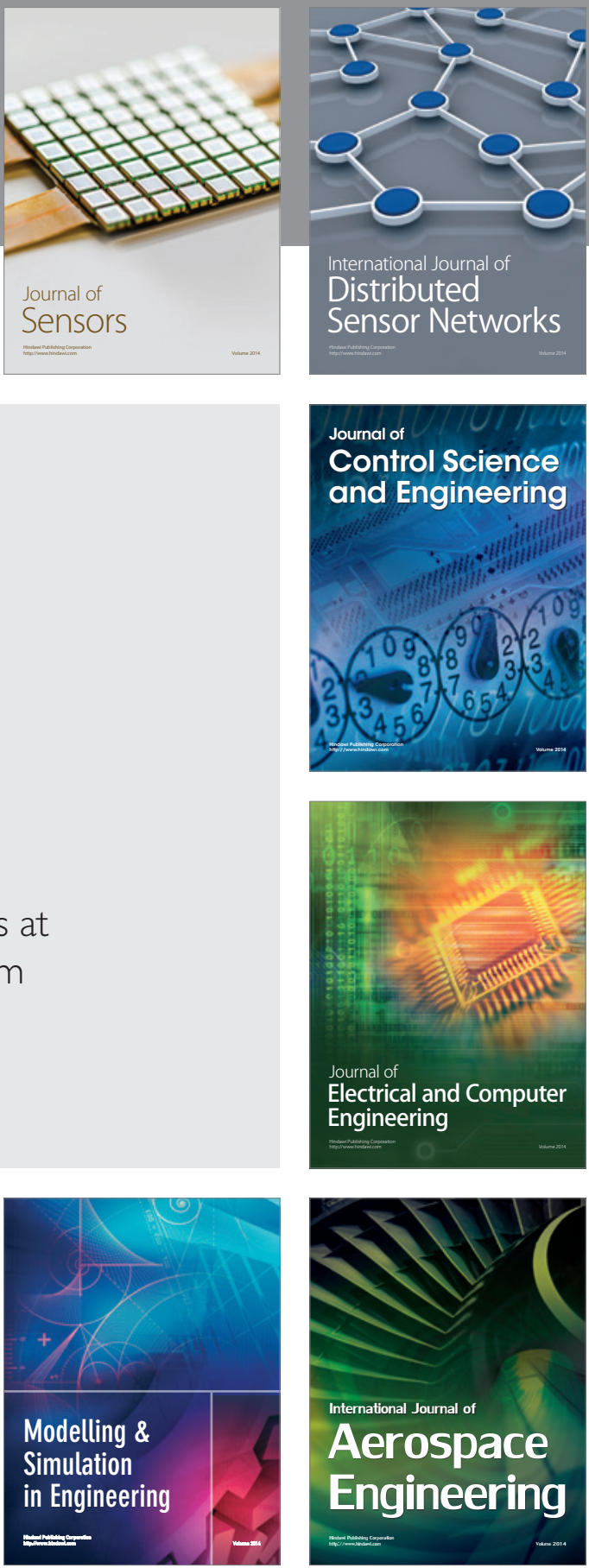

Journal of

Control Science

and Engineering
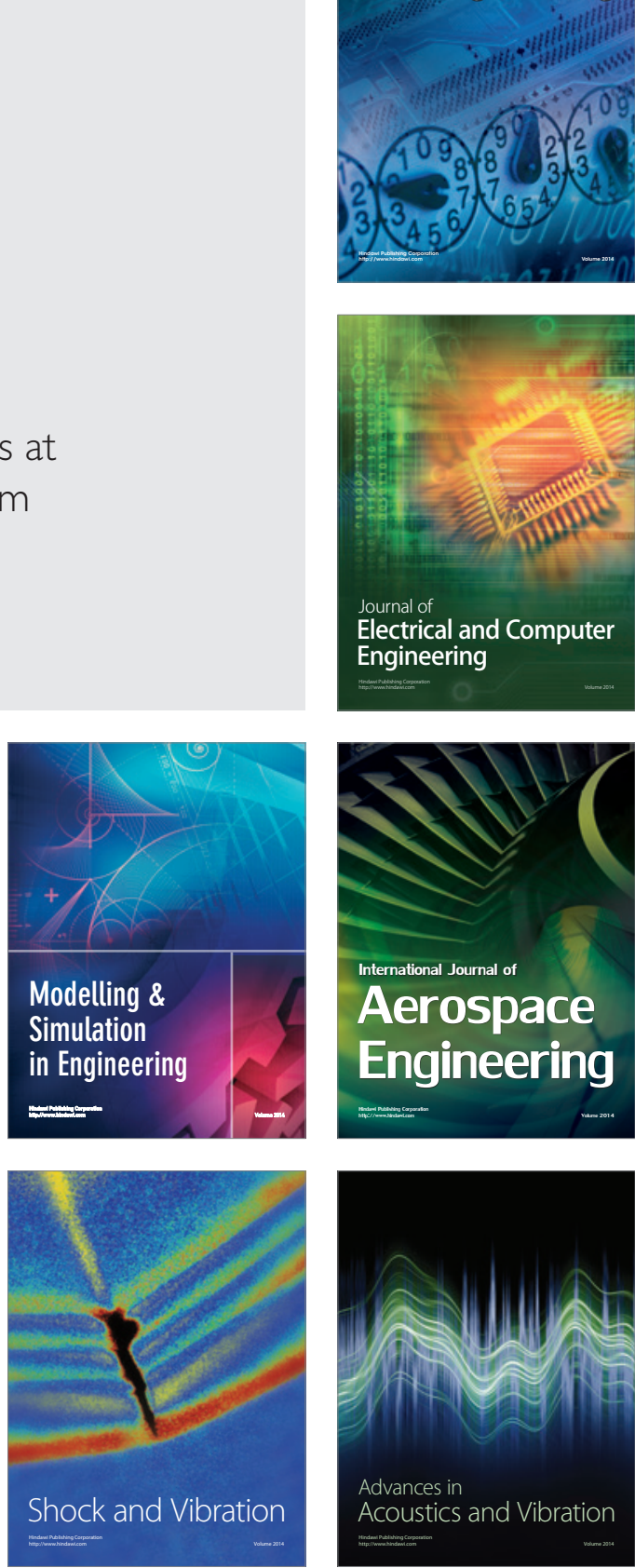